\title{
A141 IMMUNE CELL-DERIVED MICROPARTICLES IN POLYMYOSITIS/DERMATOMYOSITIS
}

Z Baka, L Senolt, J Vencovsky, H Mann, P Sebestyén Simon, A Kittel, E Buzás, G Nagy Semmelweis University, Department of Rheumatology, Hungary

\subsection{6/ard.2010.129643r}

Microparticles are recently recognised players of intercellular communication. They are involved in signal transduction, cell activation and apoptosis. Their importance is also suggested in autoimmune diseases such as rheumatoid arthritis and systemic sclerosis. We investigated the role of microparticles in polymyositis/dermatomyositis, a group of rare autoimmune diseases characterised by specific skin lesions and muscle weakness. The plasma concentration of monocyte and lymphocyte-derived microparticles of 20 patients with polymyositis/dermatomyositis and 20 healthy controls were determined by flow cytometry. The structure of microparticles was visualised by electron microscopy. Significantly elevated numbers of monocyte (CD14 positive), T lymphocyte (CD3 positive) and B lymphocyte (CD19 positive)-derived microparticles were found in the plasma samples of patients with polymyositis/dermatomyositis compared with healthy controls $(p=0.01, p=0.01$ and $p=0.06$, respectively). Furthermore, the plasma levels of monocyte and B lymphocyte derived microparticles correlated with the manual muscle strength test $(r=497, p=027 ; r=508$, $\mathrm{p}=023$; respectively). Patients with anti-Jo- 1 antibody and lung involvement had significantly higher numbers of $T$ and $B$ lymphocyte and monocyte-derived microparticles $(p=006$, $p=012$ and $p=007$, respectively, for anti-Jo-1; $p=013, p=016$ and $\mathrm{p}=025$, respectively, for lung involvement). After ultracentrifugation, CK activity could be detected only in traces in the resuspended pellet containing microparticles of healthy and 
diseased individuals. Electron microscopy revealed slightly different microparticles in the samples of patients with polymyositis/dermatomyositis. These results suggest that immune cell-derived microparticles may contribute to the inflammatory process in polymyositis/dermatomyositis; however, CK-positive, possibly muscle-derived microparticles do not seem to be present in the blood of patients with polymyositis/ dermatomyositis. 